\title{
Charged Eigenstate Thermalization, Euclidean Wormholes, and Global Symmetries in Quantum Gravity
}

\author{
Alexandre Belin ${ }^{a}, \quad$ Jan de Boer ${ }^{b}, \quad$ Pranjal Nayak ${ }^{c}, \quad$ Julian Sonner $^{c}$ \\ a CERN, Theory Division, 1 Esplanade des Particules, Geneva 23, CH-1211, Switzerland \\ ${ }^{b}$ Institute for Theoretical Physics, University of Amsterdam, \\ PO Box 94485, 1090 GL Amsterdam, The Netherlands, \\ c Department of Theoretical Physics, University of Geneva, \\ 24 quai Ernest-Ansermet, 1214 Genève 4, Switzerland
}

\begin{abstract}
We generalize the eigenstate thermalization hypothesis to systems with global symmetries. We present two versions, one with microscopic charge conservation and one with exponentially suppressed violations. They agree for correlation functions of simple operators, but differ in the variance of charged one-point functions at finite temperature. We then apply these ideas to holography and to gravitational low-energy effective theories with a global symmetry. We show that Euclidean wormholes predict a non-zero variance for charged one-point functions, which is incompatible with microscopic charge conservation. This implies that global symmetries in quantum gravity must either be gauged or explicitly broken by non-perturbative effects.
\end{abstract}

\section{INTRODUCTION}

The thermal behavior of quantum many-body systems is well understood in terms of statistical mechanics. However, developing a microscopic understanding of thermalization is a difficult problem of sustained interest. The eigenstate thermalization hypothesis (ETH) [1, 2] is a powerful framework to understand how a pure state can give rise to thermal behavior after sufficiently long times. The crux lies in the fact that individual eigenstates behave like a statistical ensemble for a large class of observables, with pseudo-random corrections that are exponentially small in the entropy. The ETH states that for simple (few-qubit) operators $O^{a}$, we have

$$
\left\langle E_{i}\left|O^{a}\right| E_{j}\right\rangle=f^{a}(\bar{E}) \delta_{i, j}+g^{a}(\bar{E}, \omega) e^{-S(\bar{E}) / 2} R_{i j},
$$

where $\bar{E}$ and $\omega$ are the mean energy and energy difference of the states $i$ and $j$, respectively. The matrix $R_{i j}$ is comprised of erratic order one numbers which statistically have zero mean and unit variance. In any given quantum system with fixed Hamiltonian, they are definite numbers that could be obtained by diagonalizing the Hamiltonian. However, for the purpose of computing few-point correlation functions of simple operators in high energy states, these microscopic details are irrelevant and it suffices to treat the $R_{i j}$ as true random variables. This randomness is tightly linked to the connection between quantum chaotic systems and random matrix theory (see 3 for a review).

New insights into the randomness of chaotic quantum systems have emerged from gravitational physics, through holographic duality [4]. If the chaotic quantum system at hand is a large $N$, strongly coupled conformal field theory (i.e. a holographic CFT), thermalization of the boundary quantum system is connected to black hole formation in the gravitational dual 5 [8]. In fact, the apparent loss of unitarity in both these processes is closely related and understanding one will help in the understanding of the other. Indeed quantum thermalization has been discussed in the context of holography for precisely this reason (see for example [9 20]).

\section{Randomness in Holography}

It has recently become clear that the low energy effective theory on the gravity side (i.e. semi-classical general relativity and its Euclidean path integral) has the potential to know quite a lot about the structure of eigenstates of the CFT Hamiltonian, perhaps much more than we had hoped for. While it has long been known that the Bekenstein-Hawking formula computes the (coarse-grained) entropy of black hole micro-states, recent progress has established that the low energy effective theory also knows something about fine structure of the microstates and their discrete nature, for example the level-repulsion of nearby eigenvalues of the Hamiltonian 21. New field configurations known as Euclidean wormholes contributing to the gravitational path integral play a crucial role in these developments. These may or may not be saddle-points [22 24].

Precisely quantifying the amount of CFT information that the gravitational path integral has access to has become one of the most pressing questions in holography. Interestingly, it has given a new perspective on the ETH: rather than viewing semi-classical general relativity as a traditional low energy effective theory that computes scattering amplitudes around the vacuum, it can be viewed as an effective theory in the sense of ETH, namely a framework for computing the correlators of simple op- 
erators on black hole microstates. In this context, simple operators should be understood as operators dual to supergravity fields. Multi-trace operators are also simple as long as $\Delta_{O} \ll N$. While there are many erratic signals in such observables that cannot be accessed through the effective theory, the moments of these signals can. This led 25] to propose a framework to describe these moments in terms of the statistics of OPE coefficients. The OPE randomness hypothesis is a generalization of ETH that states that any index of an OPE coefficient labelling a black-hole microstate can effectively be treated as a random variable. A similar approach for Haar-typical states was studied in [26].

While ensemble-averaging over quantum systems has played a prominent role in two-dimensional gravity, for example in 21, this effective description is also applicable in individual quantum systems with a fixed Hamiltonian (at least for self-averaging quantities), which will be the focus of this work. A general framework explaining this mechanism and connecting it to random matrix theory was developed in [27] (see also [28, 29]). This framework leads to random fluctuations in OPE coefficients [30.

\section{Summary of results}

In this Letter, we will discuss how global symmetries interact with the ETH, wormholes and erratic signals of quantum chaos. We start by generalizing the ETH in the presence of global symmetries. For neutral operators, we can simply apply the ETH charge sector by charge sector. This is expected from a Hamiltonian decomposed into blocks corresponding to the different charge sectors, and each individual block approximates an independent random matrix 31.

Charged operators on the other hand make different charge sectors talk to one another. We discuss two possible variants of a charged ETH, one that preserves the symmetry microscopically, the other that allows for exponentially small violations of charge conservation in the random variables 1 This second version of ETH is more relevant when viewing the ansatz as an effective theory for the simple operators, where one is agnostic about whether or not the symmetry is realized microscopically. Viewed statistically, these two ansätze give equivalent answers for low-point correlators of the simple operators in any given background. However, they differ for products of correlation functions. Most notably, we have

$$
\begin{aligned}
& \left.\left\langle O_{q}\right\rangle_{\beta}\left\langle O_{q}^{\dagger}\right\rangle_{\beta}\right|_{\text {c.p. ETH }}=0 \\
& \left.\left\langle O_{q}\right\rangle_{\beta}\left\langle O_{q}^{\dagger}\right\rangle_{\beta}\right|_{\text {c.v. ETH }} \propto e^{-S},
\end{aligned}
$$

${ }^{1}$ While inessential in what follows, note that these violations affect neutral operators as well. where c.p. and c.v. denote the charge preserving and charge violating versions of ETH, respectively.

We will show that this resonates strongly with the gravitational perspective. In the bulk, multi-boundary Euclidean wormholes can give non-zero answers for the product of charged one-point functions. Whether the answer is non-zero depends on whether the symmetry is gauged in the bulk or not. If the symmetry is gauged, then we find a vanishing answer compatible with a charge preserving ETH, where the symmetry is realized microscopically. If on the contrary the symmetry is only a global symmetry of the bulk theory, the wormhole yields a non-zero answer. This implies that charged one-point functions have a non-zero variance and thus that OPE coefficients $C_{i \bar{i} q}$ are not exactly zero, but rather fluctuate with exponentially small variance. We show that provided that the wormhole accurately captures the variance of observables, this is inconsistent with exact global symmetries in quantum gravity. To summarize, either the symmetry is gauged in the bulk, or it is broken by non-perturbative effects in $G_{N}$. This provides more evidence that global symmetries cannot exist in quantum gravity [32, 33, and offers a different perspective for AdS than that of entanglement wedge reconstruction [34, 35].

We will start by describing the charged versions of ETH, before turning to gravitational computations.

Note added: while this paper was in preparation, [36. 37] appeared which contain related results in the context of replica wormholes.

\section{THE ETH WITH GLOBAL SYMMETRIES}

In this section, we will present the form of the ETH which holds in the presence of global symmetries. For concreteness, we will take the global symmetry group to be $U(1)$, but it is straightforward to generalize to other groups. In the presence of a symmetry, the charge commutes with the Hamiltonian and we can simultaneously diagonalize both operators. It is thus natural to organize the Hilbert space in different charge sectors labelled by the eigenvalue $Q$ of the charge operator. Consider now a simple operator which is neutral under the global symmetry. For such operators, the generalization of the ETH is straightforward and we have

$$
\begin{aligned}
& \left\langle E_{i}, Q_{i}\left|O_{q=0}^{a}\right| E_{j}, Q_{j}\right\rangle= \\
& \delta_{Q_{i}, Q_{j}}\left(f^{a}\left(\bar{E}, Q_{i}\right) \delta_{E_{i}, E_{j}}+g^{a}\left(\bar{E}, \omega, Q_{i}\right) e^{-S\left(\bar{E}, Q_{i}\right) / 2} R_{i j}\right),
\end{aligned}
$$

where $f_{d}{ }^{2}$ and $g_{a}$ are smooth functions of $\bar{E} \equiv\left(E_{i}+\right.$ $\left.E_{j}\right) / 2, \omega \equiv E_{i}-E_{j}$ and $Q_{i} ; R_{i j}$ are random numbers with

\footnotetext{
${ }^{2}$ An expression for $f$ in two-dimesional CFTs is given in 38 .
} 
zero mean and unit variance; and, $S\left(\bar{E}, Q_{i}\right)$ is the microcanonical entropy in a definite charge sector $Q_{i}$. Note that this is just the usual form of the ETH charge sector by charge sector. This is intuitively consistent with expectations from random matrix theory and quantum chaos, where one treats the different blocks of the Hamiltonian corresponding to each charge sector as independent random matrices 31. (see also 39]).

The story becomes more interesting when we discuss (simple) charged operators, since they automatically make different charge sectors talk to one another. In this case, the following ansatz should hold:

$$
\begin{gathered}
\left\langle E_{i}, Q_{i}\left|O_{q}^{a}\right| E_{j}, Q_{j}\right\rangle=\delta_{E_{i}, E_{j}} \delta_{Q_{i}, Q_{j}} \delta_{q, 0} f^{a}(\bar{E}, \bar{Q})+ \\
\delta_{Q_{i}, q+Q_{j}} g^{a}\left(\bar{E}, \omega, Q_{i}, Q_{j}\right) e^{-\left(S\left(\bar{E}, Q_{i}\right)+S\left(\bar{E}, Q_{j}\right)\right) / 4} R_{i j}
\end{gathered}
$$

It is worthwhile to note that unlike the case of neutral operators, there is no diagonal term for operators that carry charge. This is in fact expected: the one-point function of a charged operator vanishes in the thermal (or grand-canonical) ensemble, which only leaves room for small off-diagonal contributions in the ETH ansatz. The function $g^{a}$ is related to the (Fourier transform of the) two-point function for the operator $O_{q}$, as we now show.

Let us consider the expectation value of $O_{q}^{\dagger} O_{q}$ in an energy eigenstate and we would like to show that this quantity has a diagonal part compatible with ETH, using only (4). To do so, we insert a resolution of the identity

$$
\begin{aligned}
& \left\langle E_{i}, Q_{i}\left|O_{q}^{\dagger} O_{q}\right| E_{i}, Q_{i}\right\rangle \\
= & \sum_{E_{j}, Q_{j}}\left\langle E_{i}, Q_{i}\left|O_{q}^{\dagger}\right| E_{j}, Q_{j}\right\rangle\left\langle E_{j}, Q_{j}\left|O_{q}\right| E_{i}, Q_{i}\right\rangle \\
= & \sum_{E_{j}} e^{-\left(S\left(E_{i}+\frac{\omega}{2}, Q_{i}\right)+S\left(E_{i}+\frac{\omega}{2}, Q_{i}+q\right)\right) / 2} \\
\times & \left|g\left(E_{i}+\frac{\omega}{2}, \omega, Q_{i}+q, Q_{i}\right)\right|^{2}\left|R_{i j}\right|^{2} .
\end{aligned}
$$

The random variables $R_{i j}$ will average out to unity upon taking the sum over $j$ since they have unit variance. Moreover, we can replace the dense sum over $E_{j}$ by an integral, namely $\sum_{E_{j}} \rightarrow \int d \omega e^{S\left(E_{i}+\omega, Q_{i}+q\right)}$, which gives

$$
\begin{aligned}
& \left\langle E_{i}, Q_{i}\left|O_{q}^{\dagger} O_{q}\right| E_{i}, Q_{i}\right\rangle= \\
& \int d \omega e^{S\left(E_{i}+\omega, Q_{i}+q\right)-\left(S\left(E_{i}+\frac{\omega}{2}, Q_{i}\right)+S\left(E_{i}+\frac{\omega}{2}, Q_{i}+q\right)\right) / 2} \\
& \times\left|g\left(E_{i}+\frac{\omega}{2}, \omega, Q_{i}+q, Q_{i}\right)\right|^{2} .
\end{aligned}
$$

All remaining functions are smooth and rapidly decaying functions of $\omega$ and $q$, so we can Taylor expand them to obtain to leading order

$$
\left\langle E_{i}, Q_{i}\left|O_{q}^{\dagger} O_{q}\right| E_{i}, Q_{i}\right\rangle \approx \int d \omega e^{\frac{\beta}{2}(\omega-\mu q)}\left|g\left(E_{i}, \omega, Q_{i}, Q_{i}\right)\right|^{2},
$$

where we defined $\beta \equiv \frac{\partial S}{\partial E}$ and $\mu \equiv-\frac{1}{\beta} \frac{\partial S}{\partial Q}$. The result (7) should be given by the microcanonical average for the operator $O_{q}^{\dagger} O_{q}$ if it is to satisfy ETH, which fixes the function $g$ and its relation to the microcanonical expectation value of $O_{q}^{\dagger} O_{q}$.

Before moving on to discuss the implications for gravitational theories, we would like to discuss another type of charged ETH ansatz, which will mildly break charge conservation. Instead of (4), consider the ansatz

$$
\begin{aligned}
& \left\langle E_{i}, Q_{i}\left|O_{q}^{a}\right| E_{j}, Q_{j}\right\rangle=\delta_{E_{i}, E_{j}} \delta_{Q_{i}, Q_{j}} \delta_{q, 0} f^{a}(\bar{E}, \bar{Q}) \\
& \quad+\quad \tilde{g}^{a}(\bar{E}, \omega, \bar{Q}, \delta Q, q) e^{-S(\bar{E}, \bar{Q}) / 2} R_{i j} .
\end{aligned}
$$

The main difference between (4) and (8) is that this second version replaces the exact charge conservation by a smooth function of $\delta Q=Q_{i}-Q_{j}$ which is rapidly decaying as a function of $\delta Q-q$. From this ansatz, one could also relate the function $\tilde{g}$ to the microcanonical two-point function as in (7) (see the supplemental material for details). We would like to emphasize that the two ansätze only differ up to exponentially small corrections and are therefore indistinguishable for simple operators.

A reason to consider such a charge-breaking ansatz is the following: if we have a set of simple operators that preserve some global symmetry but we are unsure whether the microscopic Hamiltonian truly preserves this symmetry, it is perhaps more cautious to only enforce an approximate global symmetry. This would be useful for example if one wanted to formulate an effective theory for the simple operators in high energy states.

\section{EUCLIDEAN WORMHOLES}

In this section, we compute correlation functions of charged operators in gravitational theories. We are interested in the simplest possible setup with a wormhole solution connecting two asymptotic boundaries. The simplest solution of this type arises in $\mathrm{AdS}_{3}$ when the two boundaries have negative constant curvature, hence we consider two genus- 2 surfaces at the boundary 3

The relevant gravitational low energy effective theory is given by the Euclidean action

$$
\begin{aligned}
S & =-\frac{1}{16 \pi G_{N}} \int d^{3} x \sqrt{g}\left(R+\frac{2}{\ell_{A d S}^{2}}\right)+S_{\text {matter }} \\
S_{\text {matter }} & =\frac{1}{2} \int d^{3} x \sqrt{g}\left(|\partial \phi|^{2}+m^{2}|\phi|^{2}\right) .
\end{aligned}
$$

\footnotetext{
${ }^{3}$ Note that because we are considering genus- 2 boundaries, we are not computing thermal one-point functions and their variance but rather genus-2 one-point functions. Instead of probing the variance of $C_{\bar{i} i q}$, we instead probe $C_{\bar{l} q k} C_{i j l} \overline{C_{i j k}}$. This does not affect our conclusion for global symmetries.
} 
Note that this action has a global $U(1)$ symmetry. The metric of this genus- 2 wormhole reads

$$
d s^{2}=\ell_{A d S}^{2}\left(d \tau^{2}+\cosh ^{2} \tau d \Sigma_{2}^{2}\right),
$$

where $d \Sigma^{2}$ is a constant curvature metric on the genus2 surface. This geometry is locally $\mathrm{AdS}_{3}$ and can be obtained from the hyperbolic ball $\mathbb{H}_{3}$ by taking a quotient with respect to a Fuchsian group $\Gamma$ which is a discrete subgroup of the AdS isometries.

Because the scalar theory is a free field theory and the geometry (10) is a quotient of $\mathrm{AdS}_{3}$, the two-point function on the wormhole is obtained by the sum over images. For two operators inserted on opposite boundaries (see Fig. 1), the correlation function reads [22]

$$
\left.\left\langle O_{q}\right\rangle_{g=2}\left\langle O_{q}^{\dagger}\right\rangle_{g=2}\right|_{\text {gravity }} \sim \sum_{h \in \Gamma} \frac{1}{[\cosh (h(s))]^{2 \Delta}},
$$

where $s$ is the distance between the 2 points on the boundary Riemann surface and $\Delta$ is the conformal dimension of the CFT operator dual to $\phi$. For sufficiently large $\Delta$, this sum converges which is related to the wormhole being (perturbatively) stable. The fact that this correlation function doesn't vanish has an interpretation in terms of the variance of the genus- 2 one-point function of the operator $O$, which carries global charge. As we will discuss in the next section, this has drastic consequences for global symmetries in quantum gravity.
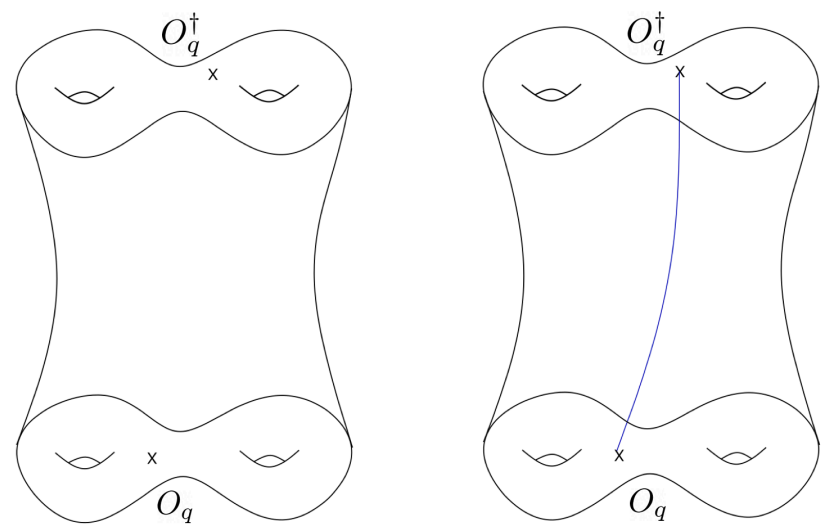

FIG. 1. A genus-2 wormhole on which we compute correlation functions. On the left, the situation where the symmetry in the bulk is not gauged. This yields a non-zero correlation function. On the right, the situation where the symmetry is gauged in the bulk. The two operators must be connected by a Wilson line for the configuration to be gauge invariant. This correlation function vanishes.

Before moving on to the consequences of such nonvanishing correlation functions for quantum gravity, we will first discuss the situation where the $U(1)$ global symmetry of the field $\phi$ is gauged. In this case, the boundaryto-boundary correlation function in the bulk is not gaugeinvariant unless the two operators are connected by a
Wilson line that propagates through the wormhole. This is depicted on the right hand side of Fig. 11. In this case, the correlation function vanishes, as already noted in [22].

The simplest way to see this is to note that in the presence of multiple boundaries, the asymptotic symmetry due to the bulk gauge field becomes one copy of the global symmetry per disconnected Euclidean boundary. In the case of our genus- 2 wormhole, the boundary global symmetry is $U(1) \times U(1)$ and the correlation function $\left\langle O_{q}\right\rangle_{g=2}\left\langle O_{q}^{\dagger}\right\rangle_{g=2}$ is charged under it (even if it is neutral under the diagonal subgroup). It must hence vanish. We therefore have 4

$$
\left.\left\langle O_{q}\right\rangle_{g=2}\left\langle O_{q}^{\dagger}\right\rangle_{g=2}\right|_{\text {gauged }}=0 .
$$

It is worthwhile to note that this does not imply that the correlation function $\left\langle\phi(x)^{\dagger} W(x, y) \phi(y)\right\rangle$ vanishes for bulk points that do not reach the boundary. Such correlation functions are perfectly fine gauge-invariant objects of the bulk theory, even if they are separated in Euclidean time (in particular, they are neutral under the $U(1) \times U(1)$ global symmetry of the boundary). Therefore, charge can propagate through the wormhole, but it is forbidden to reach the boundaries. We now turn to the discussion of global symmetries in quantum gravity.

\section{NO GLOBAL SYMMETRIES IN QUANTUM GRAVITY}

We will now show that the existence of multi-boundary Euclidean wormholes prevents the existence of exact global symmetries in quantum gravity. First, we need to discuss the difference between an exact global symmetry of quantum gravity and one that is gauged. From the CFT standpoint, both involve a graded operator algebra and exact selection rules for correlation functions: a correlation function is non-zero only if the sum of the charges of all operators vanishes. In particular, this applies to all OPE coefficient which must satisfy charge conservation

$$
C_{O_{q_{1}} O_{q_{2}} O_{q_{3}}} \propto \delta_{q_{1}+q_{2}+q_{3}, 0} .
$$

The difference between a situation where the symmetry is gauged in the bulk involves the dual of the bulk gauge

\footnotetext{
4 The same argument applies to other types of symmetries, even spacetime ones. For example, a finite temperature onepoint function is independent of Euclidean time. Therefore $\left\langle O\left(\tau_{1}\right)\right\rangle_{\beta}\left\langle O\left(\tau_{2}\right)\right\rangle_{\beta}$ must be independent of $\tau_{1}$ and $\tau_{2}$. A given wormhole solution may naively look like it gives a non-trivial $\tau_{1}-\tau_{2}$ dependence, but this will be destroyed by integrating over a family of wormhole solutions that restore time translation symmetry on both boundaries. In our situation, this integral is over the gauge field.
} 
field: a CFT current which implements the action of the global symmetry.

It is currently unknown whether a consistent CFT with a local stress-tensor can have an exact symmetry for all its correlation functions without having a current (see 34] for a detailed discussion on such issues) ${ }^{5}$ Here, we will show that the low-energy gravitational effective theory is smart enough to know that exact global symmetries are not allowed. Note that independently of whether the symmetry is gauged in the bulk, the one-point function of a charged operator must vanish on any compact Euclidean surface. In particular, we have

$$
\left\langle O_{q}\right\rangle_{g=2}=0 .
$$

This is an exact statement, and follows from the selection rule (13). We will now see that this is in conflict with the wormhole answer.

The existence of wormhole solutions in the bulk and the correlations they imply may seem puzzling at first sight, but such wormhole correlation functions have been interpreted as encoding the variance of microscopic CFT observables as the result of some coarse-graining. For one-point functions, we have

$$
\left.\langle O\rangle\langle O\rangle\right|_{\text {gravity }}=\overline{\left|\langle O\rangle_{\mathrm{CFT}}\right|^{2}}
$$

where the ${ }^{-}$notation refers to some coarse-graining involving averaging over an energy band which we will not aim to make precise here. In general, when quantities like OPE coefficients or spectral phases are erratically varying, wormhole contributions can give us the mean and variance of such signals. The central assumption that we will make is that the wormhole contribution in gravity accurately captures the CFT variance for this type of signal.

Here, this leads to a paradox. The wormhole correlation function (11) is non-vanishing, implying that the charged one-point function has some variance. But this is in direct contradiction with 14 which asserts that charged one-point functions are exactly zero. What this is showing us is that if we try to enforce an exact global symmetry in quantum gravity, the existence of Euclidean wormholes tells us that this symmetry cannot be exact, and must necessarily be broken by non-perturbative effects. It is remarkable that the low-energy gravitational effective theory and its Euclidean path integral are smart enough to know this. On the other hand, if the symmetry is gauged in the bulk, the exact microscopic selection rule is enforced by the gauge symmetry and the variance (i.e. wormhole contribution) exactly vanishes.

\footnotetext{
${ }^{5}$ In the absence of a local stress-tensor, such CFTs clearly exist: the canonical example is generalized free fields. In fact any QFT in AdS with a global symmetry will generate such a CFT.
}

Let us now make contact with the ETH ansatz in the presence of a global symmetry. We proposed two ansätze for ETH, one that exactly preserves the global symmetry and one that only approximately does. Recall that for one-boundary correlators, charged was not violated by large amounts for either ansatz and the two agreed up to exponentially small corrections. We can now see what type of theories the two different ETH describe, and when they give different answers: for multi-boundary correlation functions.

\section{DISCUSSION}

In this paper, we have presented the generalization of the ETH when there are additional global symmetries, and discussed two possible version of the ansatz: one that manifestly preserves the symmetry microscopically, and another that only preserves it for simple operators, but allows exponentially small violations of charge conservation. We then discussed a manifestation of these two scenarios for CFTs with a holographic dual in terms of Euclidean wormholes. Assuming that Euclidean wormhole computations done with the low-energy gravitational theory accurately captures moments of certain pseudo-random signals of quantum chaos in the dual CFT, we have shown that global symmetries cannot exist in quantum gravity (at least for quantum gravity in Anti-de Sitter space).

There are two possible outcomes for the fate of a global symmetry present in the low energy effective theory: it can be explicitly broken by non-perturbative effects, and we can give a lower bound on the scale of such a breaking from the gravitational action of the wormhole, namely $e^{-\ell_{A d S} / G_{N}}$ 25]. This is compatible with previous findings (see for example [40]). Alternatively, it can be gauged in which case the Euclidean wormhole computation vanishes and the symmetry is exact in the microscopic CFT description. In such a case, we cannot bound the magnitude of the gauge coupling.

It is interesting to observe that the absence of global symmetries in quantum gravity is tightly connected to the fact that low-energy observers can accurately resolve charges, in contrast with energy levels which are exponentially dense.

We conclude with some open questions. The existence of Euclidean wormholes prevents the factorization of products of CFTs on disconnected manifolds, which is inconsistent with any microscopic CFT computation. While this can sometimes originate from taking ensemble averages over microscopic theories, one would also like to understand the role of wormholes in definite unitary CFTs, and how factorization is restored. There is evidence that factorization can be restored by considering certain UV ingredients like branes, which account for "non-diagonal" elements of the quasi-random variables 
[4143. One may wonder if UV ingredients could resolve the wormhole contribution (11) and the associated tension with global symmetries in quantum gravity. The point we are making here is that in the presence of an exact global symmetry, all moments of charged one-point functions must vanish, which is not what we observe for the variance. This is irrespective of how factorization is restored.

Another avenue to consider is to understand the interplay between our results concerning energy eigenstates and typical states obtained from Haar averaging in the microcanonical window [26]. It is worth pointing out that the charge violating version of ETH is very similar to formulas one would obtain when Haar-averaging over an ensemble of states that contain several different charge sectors. It would thus naturally arise in such a context.

Taking a step back, we are proposing that a CFT framework that can encode all observables the low energy gravitational theory has access to, is a theory of OPE coefficients treated statistically. Gravitational computations give us access to (arbitrary) moments of the statistical distribution of microscopic data ${ }^{6}$ In this work, we have shown that this line of thought leads to a novel argument against global symmetries in theories of quantum gravity. It would be very interesting to connect our reasoning to the standard arguments against global symmetries coming from black hole physics. We hope to return to these questions in the future.

\section{ACKNOWLEDGEMENTS}

We are happy to thank Daniel Harlow, Nabil Iqbal, Lampros Lamprou, Raghu Mahajan, Kyriakos Papadodimas, Gui Pimentel, Gabor Sarosi, Edgar Shaghoulian and Sasha Zhiboedov for fruitful discussions. We would also like to acknowledge Island Hopping 2020 where many useful conversations on this project took place. $\mathrm{JdB}$ is supported by the European Research Council under the European Unions Seventh Framework Programme (FP7/2007-2013), ERC Grant agreement ADG 834878. This work has been partially supported by the SNF through Project Grants 200020 182513, as well as the NCCR 51NF40-141869 The Mathematics of Physics (SwissMAP).

[1] J. M. Deutsch. Quantum statistical mechanics in a closed system. Phys. Rev. A, 43:2046-2049, Feb 1991.

\footnotetext{
${ }^{6}$ An interesting challenge for this program is that the gravitational theory has access to data coming from putting CFTs on arbitrary manifolds, which in $d>2$ does not manifestly connect to the local data of the CFT (see [4]).
}

[2] Mark Srednicki. Chaos and quantum thermalization. Phys. Rev. E, 50:888-901, Aug 1994.

[3] Luca D'Alessio, Yariv Kafri, Anatoli Polkovnikov, and Marcos Rigol. From quantum chaos and eigenstate thermalization to statistical mechanics and thermodynamics. Adv. Phys., 65(3):239-362, 2016.

[4] Juan Martin Maldacena. The Large N limit of superconformal field theories and supergravity. Int.J.Theor.Phys., 38:1113-1133, 1999.

[5] Joao Aparicio and Esperanza Lopez. Evolution of TwoPoint Functions from Holography. JHEP, 12:082, 2011.

[6] V. Balasubramanian, A. Bernamonti, J. de Boer, N. Copland, B. Craps, E. Keski-Vakkuri, B. Muller, A. Schafer, M. Shigemori, and W. Staessens. Holographic Thermalization. Phys. Rev. D, 84:026010, 2011.

[7] Tarek Anous, Thomas Hartman, Antonin Rovai, and Julian Sonner. Black Hole Collapse in the 1/c Expansion. JHEP, 07:123, 2016.

[8] Tarek Anous, Thomas Hartman, Antonin Rovai, and Julian Sonner. From Conformal Blocks to Path Integrals in the Vaidya Geometry. JHEP, 09:009, 2017.

[9] Hong Liu and S. Josephine Suh. Entanglement Tsunami: Universal Scaling in Holographic Thermalization. Phys. Rev. Lett., 112:011601, 2014.

[10] A. Liam Fitzpatrick, Jared Kaplan, and Matthew T. Walters. Virasoro Conformal Blocks and Thermality from Classical Background Fields. JHEP, 11:200, 2015.

[11] Nima Lashkari, Anatoly Dymarsky, and Hong Liu. Eigenstate Thermalization Hypothesis in Conformal Field Theory. J. Stat. Mech., 1803(3):033101, 2018.

[12] Julian Sonner and Manuel Vielma. Eigenstate thermalization in the Sachdev-Ye-Kitaev model. JHEP, 11:149, 2017.

[13] Alexander Maloney, Gim Seng Ng, Simon F. Ross, and Ioannis Tsiares. Generalized Gibbs Ensemble and the Statistics of KdV Charges in 2D CFT. JHEP, 03:075, 2019.

[14] Anatoly Dymarsky and Kirill Pavlenko. Generalized Gibbs Ensemble of 2d CFTs at large central charge in the thermodynamic limit. JHEP, 01:098, 2019.

[15] Shouvik Datta, Per Kraus, and Ben Michel. Typicality and thermality in 2d CFT. JHEP, 07:143, 2019.

[16] Jan De Boer, Rik Van Breukelen, Sagar F. Lokhande, Kyriakos Papadodimas, and Erik Verlinde. Probing typical black hole microstates. JHEP, 01:062, 2020.

[17] Tarek Anous and Julian Sonner. Phases of scrambling in eigenstates. SciPost Phys., 7:003, 2019.

[18] Pranjal Nayak, Julian Sonner, and Manuel Vielma. Eigenstate Thermalisation in the conformal Sachdev-YeKitaev model: an analytic approach. JHEP, 10:019, 2019.

[19] Pranjal Nayak, Julian Sonner, and Manuel Vielma. Extended Eigenstate Thermalization and the role of FZZT branes in the Schwarzian theory. JHEP, 03:168, 2020.

[20] Phil Saad. Late Time Correlation Functions, Baby Universes, and ETH in JT Gravity. 102019.

[21] Phil Saad, Stephen H. Shenker, and Douglas Stanford. JT gravity as a matrix integral. 32019.

[22] Juan Martin Maldacena and Liat Maoz. Wormholes in AdS. JHEP, 02:053, 2004.

[23] Douglas Stanford. More quantum noise from wormholes. 82020.

[24] Jordan Cotler and Kristan Jensen. Gravitational Constrained Instantons. 102020. 
[25] Alexandre Belin and Jan de Boer. Random Statistics of OPE Coefficients and Euclidean Wormholes. 62020.

[26] Jason Pollack, Moshe Rozali, James Sully, and David Wakeham. Eigenstate Thermalization and Disorder Averaging in Gravity. 2020.

[27] Alexander Altland and Julian Sonner. Late time physics of holographic quantum chaos. 82020.

[28] Hong Liu and Shreya Vardhan. Entanglement entropies of equilibrated pure states in quantum many-body systems and gravity. 82020.

[29] Kevin Langhoff and Yasunori Nomura. Ensemble from Coarse Graining: Reconstructing the Interior of an Evaporating Black Hole. Phys. Rev. D, 102(8):086021, 2020.

[30] Alexandre Belin, Jan de Boer, Pranjal Nayak, and Julian Sonner. To appear.

[31] Daniel Kapec, Raghu Mahajan, and Douglas Stanford. Matrix ensembles with global symmetries and 't Hooft anomalies from 2d gauge theory. JHEP, 04:186, 2020.

[32] Charles W. Misner and John A. Wheeler. Classical physics as geometry: Gravitation, electromagnetism, unquantized charge, and mass as properties of curved empty space. Annals Phys., 2:525-603, 1957.

[33] Tom Banks and Nathan Seiberg. Symmetries and Strings in Field Theory and Gravity. Phys. Rev. D, 83:084019, 2011.

[34] Daniel Harlow and Hirosi Ooguri. Symmetries in quantum field theory and quantum gravity. 102018.
[35] Daniel Harlow and Edgar Shaghoulian. Global symmetry, Euclidean gravity, and the black hole information problem. 102020.

[36] Yiming Chen and Henry W. Lin. Signatures of global symmetry violation in relative entropies and replica wormholes. 112020.

[37] Po-Shen Hsin, Luca V. Iliesiu, and Zhenbin Yang. A violation of global symmetries from replica wormholes and the fate of black hole remnants. 112020.

[38] Diptarka Das, Shouvik Datta, and Sridip Pal. Charged structure constants from modularity. JHEP, 11:183, 2017.

[39] Junyu Liu. Scrambling and decoding the charged quantum information. Phys. Rev. Research, 2:043164, Oct 2020.

[40] Tristan Daus, Arthur Hebecker, Sascha Leonhardt, and John March-Russell. Towards a Swampland Global Symmetry Conjecture using weak gravity. Nucl. Phys. B, 960:115167, 2020.

[41] Andreas Blommaert, Thomas G. Mertens, and Henri Verschelde. Eigenbranes in Jackiw-Teitelboim gravity. 11 2019.

[42] Andreas Blommaert. Dissecting the ensemble in JT gravity. 62020 .

[43] Lorenz Eberhardt. Partition functions of the tensionless string. 82020 .

[44] Alexandre Belin, Jan De Boer, and Jorrit Kruthoff. Comments on a state-operator correspondence for the torus. SciPost Phys., 5(6):060, 2018. 